\title{
Gathering for God. George Brown in Oceania de Helen Bethea Gardner
}

\section{Gilles Bounoure}

\section{OpenEdition}

1 Journals

\section{Édition électronique}

URL : http://journals.openedition.org/jso/2242

DOI : $10.4000 /$ jso.2242

ISSN : 1760-7256

\section{Éditeur}

Société des océanistes

\section{Édition imprimée}

Date de publication : 15 décembre 2008

Pagination : 353-355

ISBN : 978-2-85430-012-3

ISSN : 0300-953x

Référence électronique

Gilles Bounoure, "Gathering for God. George Brown in Oceania de Helen Bethea Gardner », Journal de la Société des Océanistes [En ligne], 126-127 | Année 2008, mis en ligne le 15 décembre 2008, consulté le 24 septembre 2020. URL : http://journals.openedition.org/jso/2242 ; DOI : https://doi.org/10.4000/jso. 2242

Ce document a été généré automatiquement le 24 septembre 2020.

(c) Tous droits réservés 


\title{
Gathering for God. George Brown in Oceania de Helen Bethea Gardner
}

\author{
Gilles Bounoure
}

\section{RÉFÉRENCE}

Helen Bethea Gardner, Gathering for God. George Brown in Oceania, Dunedin, Otago

University Press, 208 p., bibliographie, index, carte, 31 ill. noir et blanc.

1 Les dernières mentions du nom de George Brown (1835-1917) dans les titres des journaux et même des revues scientifiques d'Occident (The Times, Anthropology Today, Museums Bulletin, etc.) remontent à plus de vingt ans, lorsque la presque totalité des objets ethnographiques collectés en Océanie par ce missionnaire méthodiste, conservés en désespoir de cause à l'université de Newcastle-on-Tyne, en Grande-Bretagne, fut vendue en 1985 au musée national ethnographique d'Osaka, au Japon, donnant lieu à un "scandale » et à des discussions plus ou moins savantes et parfois discutables elles aussi (Gathering: 152-153, à propos des revendications australiennes sur cette collection). Comme un article qui l'avait précédé (Gathering for God: George Brown and the Christian Economy in the Collection of Artefacts, in Michael O'Hanlon and Robert L. Welsch eds, Hunting the Gatherers, Ethnographic Collectors, Agents and Agency in Melanesia, 1870s-1930s, New York, Berghahn, 2000 : 35-54), le titre de cet ouvrage faisait attendre des investigations spécifiquement dévolues aux collectes de Brown, dont les circonstances, susceptibles de favoriser la compréhension de certains «types d'objet ", restent mal connues dans leur détail. Or, même en y joignant les pages consacrées aux enquêtes anthropologiques effectuées par le missionnaire, moins du quart de ce livre (154 pages sans les annexes) traite expressément de ces collectes et de leur devenir après la mort de Brown. Helen Gardner court vite dans son texte, mais on n'a pas lieu de se plaindre que son ouvrage, bref, alerte, incisif, dépasse largement son titre, qu'on pourrait dire « heureusement » trompeur. 
2 Pour qui lirait cet ouvrage au rebours de son sommaire, ne décrivant les collectes de Brown et leur devenir que dans le dernier chapitre et la conclusion, Helen Gardner ne laisse entrevoir aucun espoir de révélations ou même de précisions utiles sur ces objets provenant soit d'achats du missionnaire auprès des autochtones, soit d'échanges, soit de dons recueillis sur place, soit encore de cadeaux de collègues éloignés. Décevante en même temps qu'éclairante sur ce sujet, son enquête dans l'ensemble des écrits publics et privés laissés par Brown (à la Mitchell Library pour ces derniers) met au jour une personnalité subtile, attentive à cloisonner ses multiples activités (religieuses, politiques, «scientifiques ", sanitaires, etc.), quitte à les fondre ponctuellement quand il lui semblait nécessaire, et sensible aux soupçons que pouvait susciter sa quête effrénée de curios, jusqu'à laisser le moins de traces possibles sur la formation de son «musée » personnel. Il n'était pas le seul. Dans ses allers et retours entre Sydney et la Nouvelle-Bretagne, le John Wesley, navire de la mission méthodiste, emporta plus d'une fois des objets qu'expédiaient James Cockerell, brièvement associé à Brown (pp. 55-56, pp. 133-134), le capitaine Farrell, beau-frère et associé de Parkinson, et quelques autres, et qui ne sont pas mieux connus aujourd'hui, tout en constituant les fleurons des principaux musées d'Australie.

3 Le grand intérêt de ce livre, qui ne concerne que marginalement «l'histoire des collections ", est d'offrir une véritable plongée dans ce qu'on a pu nommer l'ethnologie missionnaire. Helen Gardner ne manque pas d'observer qu'elle est elle-même issue de milieux méthodistes - comme beaucoup de scientifiques australiens ou néo-zélandais et que la christianisation des sociétés océaniennes est achevée de longue date, tandis que pour beaucoup de spécialistes du Pacifique la figure du missionnaire est devenue, au mieux, matière à quolibets. L'analyse qu'elle livre des déterminations des méthodistes, de leurs divers concurrents présents en Océanie (la Melanesian Mission et la London Missionary Society dans les îles relevant peu ou prou de la domination britannique, les catholiques ailleurs, en attendant les Luthériens) met en évidence la position originale de Brown, qu'il devait certainement à son éducation, mais qu'il n'a cessé d'approfondir et de consolider entre sa conversion en 1855 et sa cessation partielle d'activité en 1908.

Brown n'était pas un représentant parmi d'autres du méthodisme en mission. Autodidacte, il resta intimement marqué par l'unitarisme de son père, une autre confession " hétérodoxe » britannique mais vigoureusement rationaliste et bien reçue, quant à elle, dans les milieux scientifiques, tout en devant au piétisme wesleyen de son oncle pasteur en Nouvelle-Zélande sa conversion, sa carrière et sa respectabilité. Un mélange (assez instable, démontre Helen Gardner) d'ambitions personnelles de tous ordres, de convictions religieuses parfois mises à rude épreuve, de désirs de peser sur l'histoire locale ou mondiale, une recherche également manifeste de reconnaissance personnelle expliquent l'énergie forcenée de ce "diable d'homme ", multipliant les rôles, compensant l'un par l'autre (la collecte ethnographique pouvant consoler des échecs missionnaires, lit-on p. 147), et conscient jusqu'à la mégalomanie de sa position de "pionnier", d'expert du Pacifique et d'autorité inspirée ou «missionnée », au moment où l'Océanie, nouvelle proie des impérialismes européens, allait basculer définitivement dans le jeu de l'Occident puis de la " mondialisation ».

5 Si tel est à peu près le cadre psychologique, cette rubrique ne suffirait pas à énumérer toutes les situations excellemment étudiées par Helen Gardner où ce missionnaire pétri de contradictions, et tirant certainement de leur antagonisme un surcroît d'énergie, a 
trouvé à afficher des apparences d'équilibre ou d'efficacité. Ainsi du racisme. Dans une série d'articles sous pseudonymes, Brown, coopté par les instances australiennes de son Église après deux décennies de "travail sur le terrain ", entreprenait à partir de 1881 de défendre auprès d'un large public la supériorité à tous points de vue, religieux, politique, anthropologique même, d'une mainmise britannique sur toutes les parties encore à prendre de l'Océanie : «We, as a people, with all our faults [...] do yet govern native races better than any other European race ", etc. (p. 99). Ce racisme généralisé ne heurtait en rien ses certitudes théologiques, mais quand il s'agissait des sauvages, des cannibales, il le repoussait avec force, y compris sous ses variantes évolutionnistes prétendant distinguer divers degrés de développement ou de civilisation : n'avait-il pas démontré que le plus invétéré cannibale pouvait devenir le plus sincère et le meilleur chrétien en quelques mois? D'où son refus de mesurer des capacités crâniennes, comme le lui demandaient les anthropologues européens avec lesquels il se flattait de correspondre, eux aussi pétris sans doute des meilleures intentions, et d'autres contradictions.

6 L'« humanité » contradictoire de Brown et de tous ceux qu'il a côtoyés, combattus, convertis ou repoussés, celle qui ne le fut pas moins des milieux culturels où il a vécu (de l'Angleterre divisée en « sectes non-conformistes » à l'Australie à la fois démocrate, blanche et raciste), ce livre l'explore à merveille, rapidement, avec l'esprit voulu. Qu'en conclure ? La lecture de l'ouvrage est certainement à recommander à tous les historiens se souciant de l'occidentalisation et de la christianisation du Pacifique, où le rôle de Brown reste probablement sous-estimé, alors qu'il est pleinement reconnu, dit Helen Gardner, dans l'actuelle Papouasie Nouvelle-Guinée. Mais de l'œuvre de Brown, c'est-àdire, hormis les archives qu'elle a explorées, une quinzaine d'articles et surtout ses deux «classiques", "George Brown, D.D. Pioneer-Missionary and Explorer, an Autobiography, A Narrative of Forty-Eight Years' Residence and Travel in Samoa, New Britain, New Ireland, New Guinea, and the Solomon Islands » (1908)et «Melanesians and Polynesians: Their Life-Histories Described and Compared» (1910), qu'en retenir aujourd'hui?

7 Le livre d'Helen Gardner représente sans doute un préalable indispensable à l'utilisation de ces documents, selon les connaissances et les normes scientifiques actuelles. Elle y multiplie les mises en garde, signale l'insensibilité du futur ethnographe aux aspects de la vie maori qu'il n'a pu manquer d'observer en cinq ans de séjour en Nouvelle-Zélande, son incompréhension des circuits traditionnels d'échange où il est venu brutalement s'insérer avec ses teachers, en établissant sa mission aux îles du duc d'York en 1875, conduisant à un scandaleux massacre sous ses ordres en $1878^{1}$, pointe sa duplicité à l'égard des règles mêmes de ces échanges dont il a visiblement tenté de profiter outrageusement, le caractère "arrangé » et trompeur de ses prétendues photos ethnographiques, les lacunes trop manifestement étudiées de ses publications et de ses écrits intimes... Autant de « caveat » extrêmement utiles, dans un ouvrage à peu près irréprochable (sauf pour de menus détails de prosopographie ou de bibliographie souvent faciles à rétablir, "Neu-Guniea Kompagnie» p. 98, «Henry Gigholy », p. 144 et p. 191, pour Enrico H. Giglioli, conservateur du Museo Pigorini de Rome, JSO 1, 1989, lire $n^{\circ} 88 . .$. ).

8 Si cette personnalité et cette œuvre empêtrées de contradictions inspirent dans leur ensemble des sentiments mêlés, certains détails, suggère Helen Gardner, devraient éveiller quelque sympathie. C'est ce que montre, parmi d'autres, cette anecdote qu'elle 
rapporte sans y attribuer peut-être beaucoup d'importance: s'étant mis à la photographie peu après son arrivée en Nouvelle-Bretagne, Brown fut tout surpris, le 6 décembre 1875, après avoir développé la vue qu'il venait de prendre d'un chef de la côte ouest de la Nouvelle-Irlande, et l'avoir fait admirer à son « sujet » et à ses amis, de se voir réclamer du tabac (Autobiography, pp. 136-137). Le missionnaire n'y a vu que la cupidité insatiable de ses hôtes, mais il y a certainement lieu aujourd'hui d'explorer en détail ce qu'il n'avait pas compris, les "terms of the reciprocity that accompanied all displays of economics, spiritual or technological ability in the region " (Gathering, p. 61). Dans une recherche récente (Dédommager le désir, Terrain 32, 1999 : 5-20), Monique JeudyBallini a montré la persistance dans une société christianisée de longue date, les Sulka, de cette compensation due non pas au talent ou à ses productions remarquables, mais aux manifestations d'admiration qu'ils suscitaient. Lorsque George Brown rapporte qu'il s'est vu à nouveau réclamer des cadeaux par ses spectateurs parce qu'il a joliment tiré un pigeon en Nouvelle-Irlande (Autobiography, p.137) ou parce qu'il a gracieusement relacé ses bottines après un bain en pays Tolai (ibid: 205), il semble qu'un siècle après la parution de son livre majeur il reste encore quelques notations valables à tirer de son ethnologie missionnaire si discutable par ailleurs.

\section{NOTES}

1. Au début d'avril 1878, un pasteur fijien, deux teachers et un "acolyte » de même origine, installés par George Brown, entreprennent de répandre « la bonne parole » dans l'intérieur de la péninsule de la Gazelle, au nord de la Nouvelle-Bretagne. Dès la nouvelle de leur massacre, Brown, s'appuyant sur les trafiquants blancs des environs, organise une expédition punitive, qui fait de 50 à 100 morts et incendie des villages entiers. C'est la politique ailleurs employée par la marine britannique, mais ici par un missionnaire, et sans troupes de marine. Helen Gardner consacre tout un chapitre («Murderous and vile cannibals», pp. 65-84) à cet épisode confirmant le jugement du haut-commissaire britannique pour le Pacifique occidental ayant eu à connaître de cette affaire, Arthur Gordon, assimilant ce type de missionnaire aux «évêques politiques du Moyen Âge ». La collecte de fonds auprès des Samoans et sans doute aussi dans l'archipel Bismarck est un autre aspect obscène de cette entreprise missionnaire, que suggère assez nettement cet ouvrage. 